\title{
"It's all in your head": Managing catastrophizing before it becomes a catastrophe
}

\author{
Ashley R. Cox, MD, MSc, FRCSC \\ Department of Urology, Dalhousie University, Halifax, NS, Canada
}

Cite as: Cox AR. "It's all in your head": Managing catastrophizing before it becomes a catastrophe. Can Urol Assoc J 2021;15(10):332. http://dx.doi.org/10.5489/cuaj.7592

See related article on page 326

I n medicine, "catastrophizing" is defined as an exaggerated, negative "mental set brought on during actual or anticipated painful experiences," and is one of the most important psychological predictors of pain experience. ${ }^{1}$ Catastrophizing is made up of three dimensions: helplessness ("It's awful and I feel that it overwhelms me"), rumination ("I can't stop thinking about how much it hurts"), and magnification ("I worry that something serious may happen"). ${ }^{2}$ Perhaps this is why some doctors erroneously and detrimentally inform patients that their pain is, "All in their head"?

The significance of catastrophizing behavior in patients with pelvic pain must be highlighted, as it has been linked to worsening pain severity, development of depression and anxiety, increased healthcare expenditure, and even early death in various patient populations. ${ }^{3}$ Several cross-sectional studies have identified an association between catastrophizing, severity of pain, and decreased pain-related quality of life in patients with pelvic pain. ${ }^{3,4}$

In this issue, Crawford et al sought to evaluate the directionality of the catastrophizing-pain relationship in woman with interstitial cystitis/bladder pain syndrome (IC/BPS). ${ }^{5}$ In this novel study, the results confirmed the authors' hypothesis that increased catastrophizing preceded increased pain over a 12-month period, while early increases in pain did not precede increased catastrophizing at a later date. Interestingly, when looking at the dimensions of catastrophizing, the results were mixed. Early changes in magnification predicted later changes in pain, while early changes in pain predicted later change in rumination. Helplessness, however, had a bidirectional relationship with pain, where early increases in helplessness predicted later increases in pain and early increases in pain predicted increased helplessness in the future. It was concluded that helplessness likely plays a key role in catastrophizing and worsening pain severity.

Like any study, limitations exist. This data is self-reported. The study population was made up of primarily cisgender white women with a long history of IC/BPS (mean years since diagnosis 7.95 at time 1) seen in tertiary centers; therefore, external validity of the results may be poor. Lastly, there was a fairly high attrition rate from time 1 (0 months) to time 3 (12 months).

Despite these limitations, the study results are important, as this data raises awareness of catastrophizing behaviors in this patient population. Catastrophizing likely contributes to the challenge of successfully treating IC/BPS, which leads to frustration for many patients and urologists. These results suggest a role for mental health supports, such as cognitive behavioral therapy (CBT), early on in the treatment of IC/BPS for patients who exhibit catastrophizing behavior and feelings of helplessness. CBT has been shown to be effective in patients with chronic pain ${ }^{6}$ but is yet to be thoroughly studied in IC/BPS. Hopefully, future research will move forward in this direction. The challenge then, would be focused on obtaining adequate access to mental health support for patients with IC/BPS.

Competing interests: The author does not report any competing personal or financial interests related to this work.

\section{References}

1. Sullivan MJ, Thorn B, Haythornthwaite JA, et al. Theoretical perspectives on the relation between catastrophizing and pain. Clin J Pain 2001;17:52-64. https://doi.org/10.1097/00002508-200103000-00008

2. Quartana PJ, Campbell CM, Edwards RR. Pain catastrophizing: a critical review. Expert Rev Neurother 2009;9:745-58. https://doi.org/10.1586/ern.09.34

3. Chen A, Argoff C, Crosby E, et al. Chronic pelvic pain patients demonstrate higher catastrophizing in association with pelvic symptoms and comorbid pain diagnoses. Urology 2021;150:146-50. https://doi.org/10.1016/i.urology.2020.06.055

4. Sewell M, Churilor L, Mooney S, et al. Chronic pelvic pain - pain catastrophizing, pelvic pain and quality of life. Scand J Pain 2018;18:441-8. https://doi.org/10.1515/sppain-2017-0181

5. Crawford A, Muere A, Tripp DA, et al. The chicken or the egg: Longitudinal changes in pain and catastrophizing in women with interstitial cystitis/bladder pain syndrome. Can Urol Assoc J 2021;15:326-31. http://dx.doi.org/10.5489/cuai.7106

6. Wetherell JL, Afari N, Rutledge I, et al. A randomized, controlled trial of acceptance and commitment therapy and cognitive behavioral therapy for chronic pain. Pain 2011;152:2098-2107. https://doi.org/10.1016/i.pain.2011.05.016

Correspondence: Dr. Ashley R. Cox, Department of Urology, Dalhousie University, Halifax, NS, Canada; ashleycox@Dal.Ca 